\title{
Application of Wavelet and Wiener Filtering Algorithm in Image De-Noising
}

\author{
Yuanmei Wang, Tao Li \\ School of Electronics and Information, Yangtze University, Jingzhou, China \\ Email: yuanyzq@163.com
}

Received 7 January 2016; accepted 22 January 2016; published 26 January 2016

Copyright (C) 2016 by authors and OALib.

This work is licensed under the Creative Commons Attribution International License (CC BY).

http://creativecommons.org/licenses/by/4.0/

c) (i) Open Access

\begin{abstract}
Image is often easily polluted by noise in the process of image processing, so image de-noising is an important step in the field of image processing. Based on the wavelet threshold de-noising algorithm, an improved image de-noising algorithm based on wavelet and Wiener filter is proposed in this paper, which can effectively reduce the Gaussian white noise. Firstly we use wavelet soft threshold to reduce noise, then use Wiener filter to process the image and get the valuation of the image. Experimental results show that the proposed algorithm on image de-noising not only can effectively suppress Gaussian white noise, but also can well retain the details of image edges.
\end{abstract}

\section{Keywords}

Image De-Noising, Threshold Function, Wavelet Soft Threshold, Wiener Filtering

Subject Areas: Image Processing

\section{Introduction}

In the process of image processing, image will be brought certain influence in quality when the image is in transmission and transformation, which mainly displays in image fuzzy, noise etc. In order to reduce the noise to improve the quality of the images, so the research of de-noising [1]-[3] has very important significance, it is also the focus of the image processing research. Classic de-noising method [4]-[7] is in the spatial domain, and it also can be in frequency domain. But these two kinds of de-noising method are easy to cause contradiction, although the noise is suppressed, the details information of the image edge is lost, so it is easy to cause the image blur. Because the noise spectrum and the image spectrum are easily overlapped, therefore image de-noising is a more difficult task for this kind of image with Gaussian white noise.

Wiener filter is as a kind of adaptive linear filter, it can effectively suppress noise and protect the image edge, so it is widely used in image processing. For the detail resolution is poor, it is easy to cause details information of image to be lost and damaged such as fine lines, corner details and other important information for the kind of 
bad solution image. Wavelet analysis [8] has good localization properties and the characteristics of multi-resolution analysis in time domain and frequency domain at the same time, and it can separate the signal and noise effectively, it satisfies the requirement of various de-noising such as low-pass, high-pass, random noise. Comparing with the traditional de-noising method, it has the incomparable advantages and it becomes an important tool for signal analysis. Using wavelet transform for signal de-noising, it can ensure no damage to the signal at the same time of the de-noising. Based on the above analysis, an improved wavelet and Wiener filtering algorithm in image de-noising is put forward in this paper. The method makes full use of the phase invariability of the stationary wavelet transform, fully considering the correlation of wavelet coefficient, and it gets good effect on image de-noising processing.

\section{Basic Principle of Image De-Noising Base on Wavelet Transform}

Wavelet transform [9]-[13] has local analysis characteristics in time domain and frequency domain, which is another major breakthrough after the Fourier transform. It is a kind of local analysis method whose window area is stable and shape is variable. There are high frequency resolution and low time resolution in the low frequency, and high time resolution and low frequency resolution in the high frequency, it can satisfy the adaptive changes of the time-frequency window. In wavelet de-noising methods, there are three main categories: max amplitude detection method, wavelet threshold de-noising method and shielding de-nosing method. Max amplitude detection method is one of the most commonly methods, Wavelet transform generated a few large amplitude wavelet coefficients, but the noise after wavelet transform generated many small amplitude wavelet coefficients which distributed throughout almost the whole wavelet domain. So we can separate the signal and noise by setting a threshold. How to select the wavelet coefficient is the key of wavelet threshold de-noising method. The absolute value of wavelet coefficients is a local measurement, which is as independent variable. Setting a threshold value $\mathrm{T}$, if the absolute value of wavelet coefficients is less than the threshold $\mathrm{T}$, these wavelet coefficients can be as noise, marked as " 0 "; if the absolute value of wavelet coefficients is larger than the threshold $\mathrm{T}$, these wavelet coefficients should be updated by subtracting T. According to the selection of the threshold, different threshold functions reflect different processing methods and estimation methods. The common threshold function is mainly divided into soft threshold function and hard threshold function. Soft threshold function [12] can be represented by formula (1), where $P$ as the wavelet coefficients, $T$ as the threshold.

$$
P_{1}= \begin{cases}\operatorname{sgn}(p)(|p|-T), & |p| \geq T \\ 0, & |p|<T\end{cases}
$$

Hard threshold function [13] as the following formula (2):

$$
P_{1}= \begin{cases}p & |p| \geq T \\ 0 & |p|<T\end{cases}
$$

The image as a two-dimensional digital signal, wavelet transform especially orthogonal wavelet transform has a strong data correlation. After wavelet transform, the energy of image in frequency domain can concentrate in some large wavelet coefficients, and the energy of noise is distributed in the whole wavelet domain. Analyzing and processing of image wavelet coefficient, the corresponding image de-noising algorithm can be got. The process of image wavelet threshold de-noising is shown in Figure 1, Wavelet threshold de-noising algorithm is as followings:

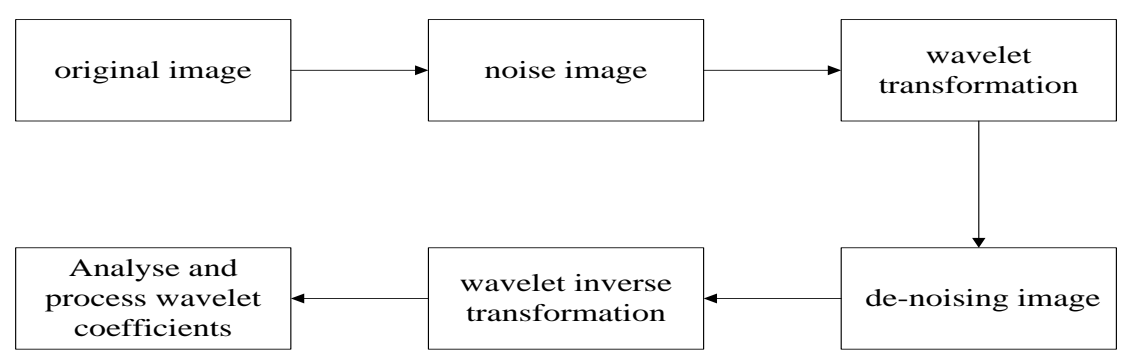

Figure 1. Process of image de-noising base on wavelet transform. 
Step 1: add some noise to the original image, then get noised image;

Step 2: noised image by wavelet transform, get wavelet coefficients;

Step 3: preserve the wavelet large coefficients in low frequency; and process the wavelet small coefficients in high frequency by threshold processing;

Step 4: process the processed wavelet coefficients, then make wavelet inverse transformation and reconstruct the original image, finally get de-noising image.

Among these steps, how to select threshold and threshold function is the core of the wavelet threshold de-noising algorithm in step 3. Noised image has wavelet transform, noise reflects the smaller coefficients in high-frequency, and image details also reflect the larger coefficients in high-frequency. Choosing appropriate threshold and threshold function are very important, if the coefficients are smaller the threshold, then these coefficients are seen as noise. So suppressing Gaussian white noise at the same time, we can keep image edge details.

\section{Wiener Filtering}

Wiener filtering [14]-[16] is a kind of adaptive filtering with minimum mean square error criterion, it is also a kind of method for the optimal linear filtering. When the signal and noise are acting on the system simultaneously, the Wiener filter is designed as far as possible to reproduce the input signal under the minimum mean square error criterion in output, the noise in the output can be suppressed. In image processing, according to the autocorrelation function of image signal and the observed value of the output, seeking for the unit impulse response of the filter with minimum mean square error, then make optimal estimation for input signal, according to the image of local variance to adjust the output value of the filter, the principle of Wiener filtering is applied to image de-noising based on wavelet transform, which can obtain good de-noising effect.

Wiener filtering has the advantages of small calculation and good noise effect, so it has been used widely. Many efficient de-noising algorithms are based on the principle of Wiener filtering, whose purpose is to restore the original image and reach the minimum mean error with the original image.

\section{Wavelet and Wiener Filtering Algorithm in Image De-Noising}

Because wavelet transform has the feature of orthogonality, Gaussian white noise with zero as mean and variance as $\sigma_{n}^{2}$ are the same in the wavelet domain and time domain. Supposing the wavelet coefficients of noised image as $G(i, j), M(i, j)$ as the wavelet coefficients of the original image, $N(i, j)$ as Gaussian white noise with "0"as mean and " 0.1 " as standard variance. Then $G(i, j)$ can be expressed as following formula (3).

$$
G(i, j)=M(i, j)+N(i, j)
$$

After wavelet transformation, then can get $g(i, j)=m(i, j)+n(i, j)$. In formula (3), $g(i, j)=W^{*} G(i, j)$, $m(i, j)=W^{*} M(i, j), n(i, j)=W^{*} N(i, j)$, here $W$ is transformation matrix of wavelet transform.

The wavelet coefficients $n(i, j)$ also has the same Characteristic as in the wavelet domain.

Because the image signal and noise are uncorrelated, the variance of wavelet coefficient of image signal can be got by formula (4).

$$
{\stackrel{\Lambda}{\sigma_{i, j}}}^{2}=E\left\{m(i, j)^{2}\right\}=\max \left(E\left\{g(i, j)^{2}\right\}-\sigma_{n}^{2}\right)
$$

In formula (4), $E\left\{g(i, j)^{2}\right\}$ can be got by calculating the sum of squares of wavelet coefficients and Theirs mean value in filter window $R$, estimating values $m(\hat{i}, j)$ of Wiener filtering can be got by using $g(i, j)$ and its domain, the specific calculation process such as formula (5).

$$
E\left\{g(i, j)^{2}\right\}=\frac{1}{N} \sum_{(a, b \in R)} g(i+a, j+b)^{2}
$$

In formula (5), $N$ as the total number of pixels in filter window $R$. Image signal can be estimated according to formula (6).

$$
m(i, j)=\frac{\stackrel{\Lambda}{\sigma_{i, j}^{2}}}{\sigma_{i, j}^{2}+\sigma_{n}^{2}} \times g(i, j)
$$


The processes of image de-noising based on wavelet and Wiener filtering are as followings:

Step 1: the noised image has three layers of wavelet decomposition with the symmetric wavelet "sym 4"; then get corresponding wavelet coefficients;

Step 2: process wavelet coefficients by soft threshold method with formula (1); according to the unbiased risk estimation criterion, find the risk value of wavelet coefficients, selected minimal risk value as value $T$.

Step 3: calculate estimated value of image signal with Wiener filtering formula (6).

Step 4: have wavelet inverse transform for image, then get de-noise image and estimate value of image.

\section{Experiment Results and Analysis}

\subsection{Experiment Results}

In this paper, the experiment is realized by program in Matlab, choosing image Lena $(256 \times 256)$ as the original image shown in Figure 2. First of all, adding Gaussian noise with "0" as mean and "0.1" as standard variance to the original image, which is shown in Figure 3. Then the noised image has wavelet decomposition, choosing "sym4" as wavelet base, decomposition layer as 3, threshold function respectively using soft threshold function and hard threshold function. After processing, the de-noised experimental results of noised image can be obtained by simulation, which are shown in Figures 2-7 respectively.

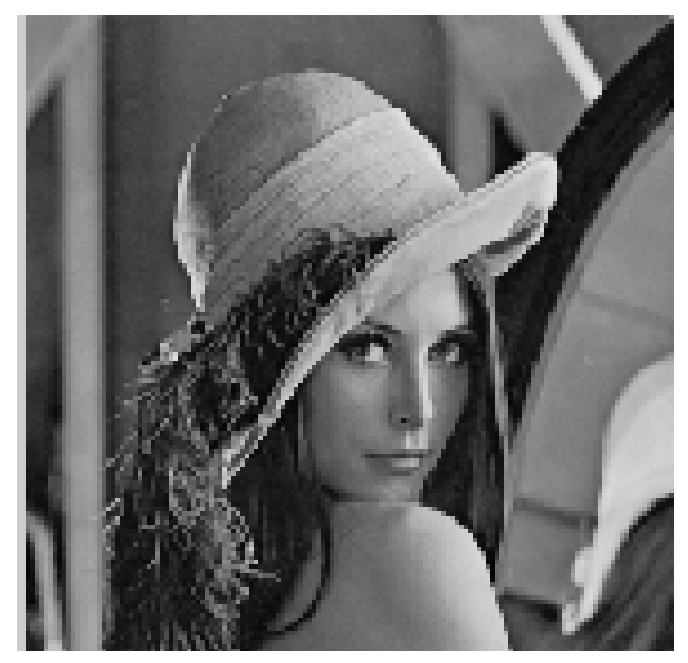

Figure 2. Original image.

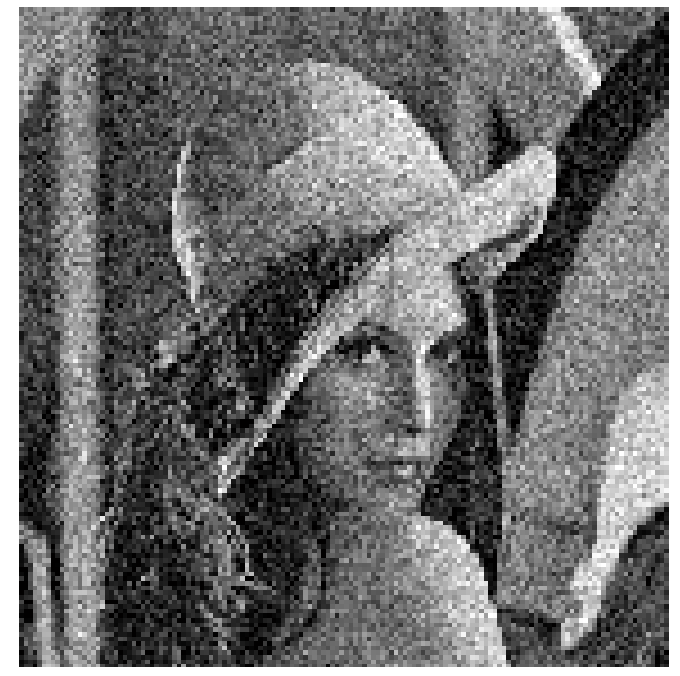

Figure 3. Noised image. 


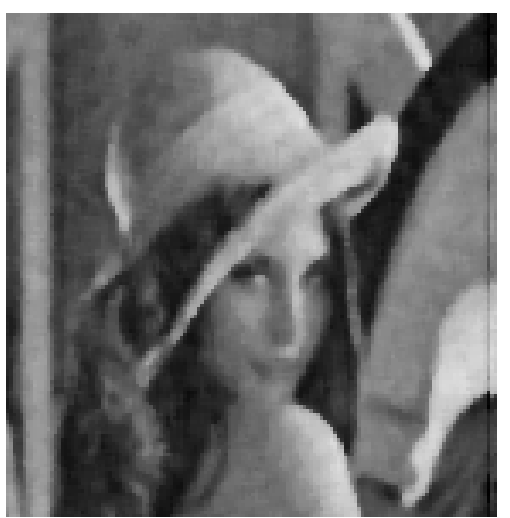

(a)

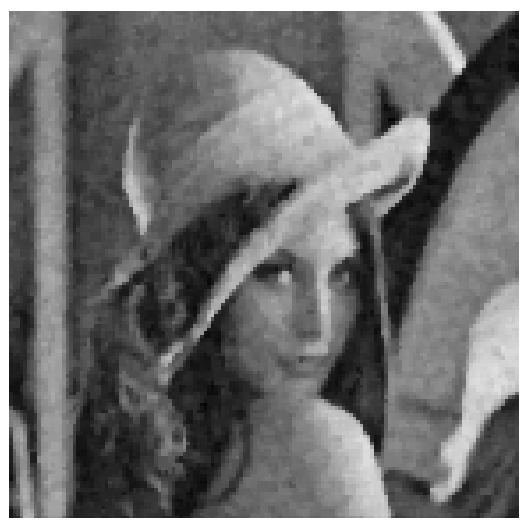

(b)

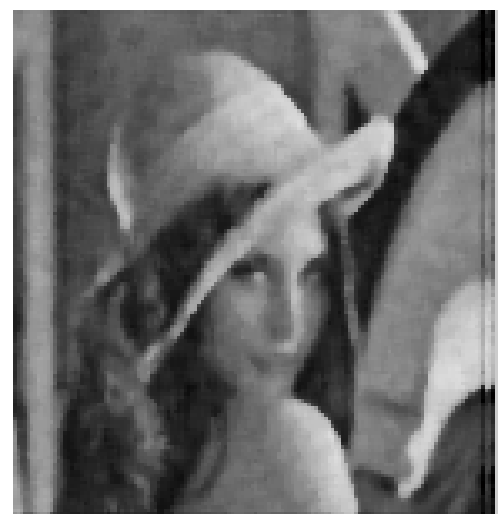

(c)

Figure 4. Results of median filtering. (a) $3 \times 3$ template; (b) $5 \times 5$ template; (c) $7 \times 7$ template.

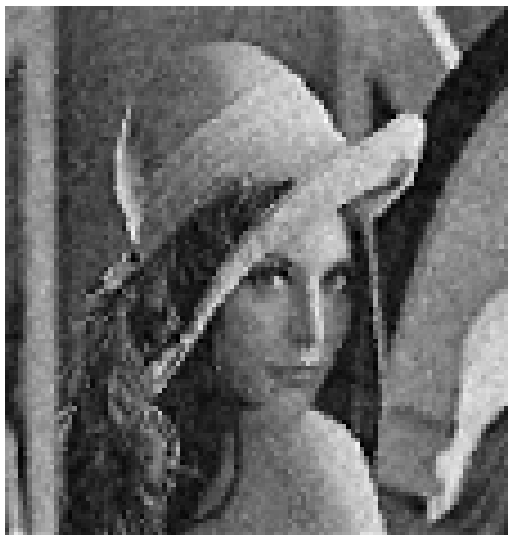

(a)

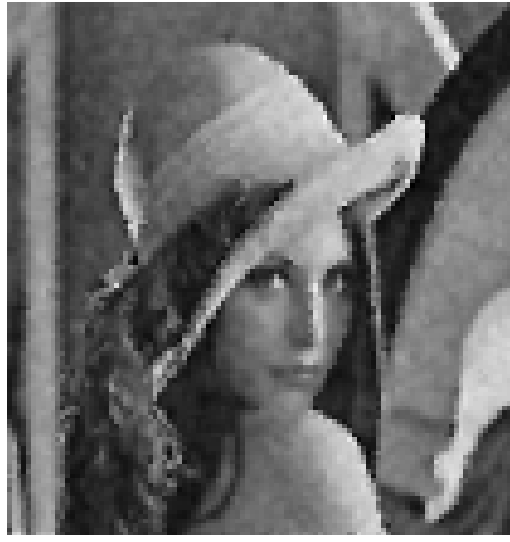

(b)

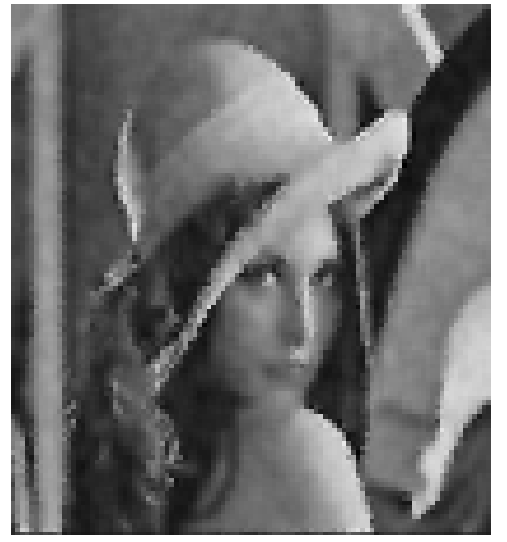

(c)

Figure 5. Results of wiener filtering. (a) $3 \times 3$ template; (b) $5 \times 5$ template; (c) $7 \times 7$ template.

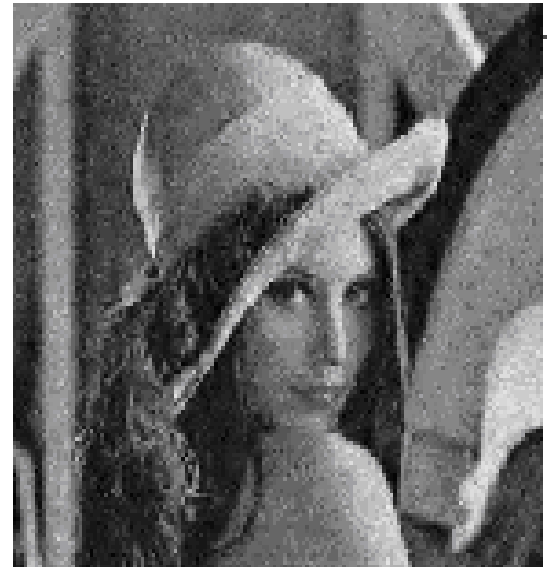

Figure 6. Results of wavelet threshold filtering.

Median filtering is a typical non-linear filtering. After median filtering, the de-noising image will occur the problem of the fuzzy edge which is shown in Figure 4. The size of template has great influence on the effect of de-noising. So in the processing of median filtering, choosing a suitable size of template is a key step. Wiener filter is an optimal linear filter, the image with Gaussian noise is processed, the better effect of de-noising is got, but the image is more blurry, the result is shown in Figure 5. The result of the wavelet threshold de-noising is 


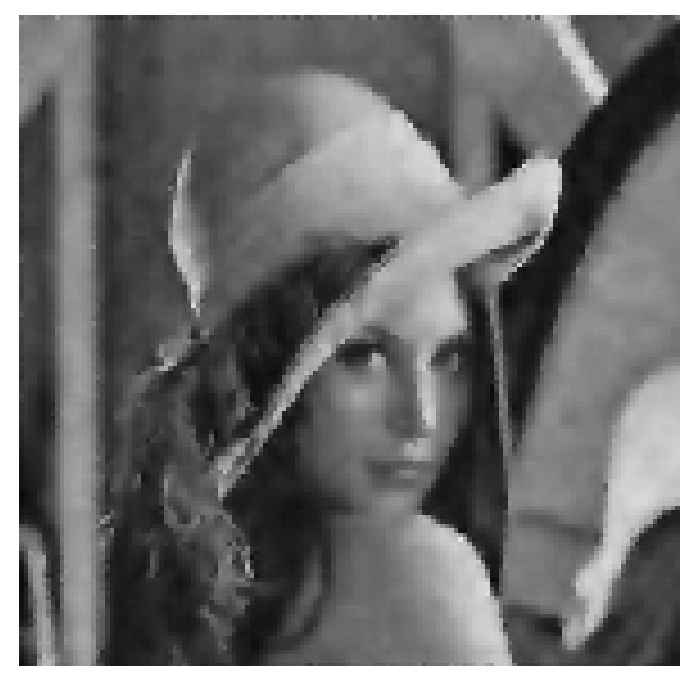

Figure 7. Results of wavelet and wiener filtering.

shown in Figure 6 when selecting suitable threshold. It does not occur the burr, but the effect of de-noising is not the best. With the proposed method of wavelet and Wiener filtering de-noising, the effect of image de-noising is better shown in Figure 7, and the edge and the outline of the image are clearer than others.

\subsection{Anti-Noise Analysis}

In this paper, PSNR and MSE are as comprehensive evaluation criteria of an algorithm. Generally, PSNR, namely Peak Signal to Noise Ratio, its unit is dB; MSE is Mean Square Error which is a feasible measure for mean error, MSE evaluates the variety of data. The value of MSE is the less, the experimental data is more precise. The PSNR and MSE are expressed as following formula (7) and formula (8) respectively.

$$
\begin{gathered}
P S N R=10 \lg \frac{M \times N \times 255^{2}}{\sum_{m=0}^{M} \sum_{n=0}^{N}\left[f(m, n)-f^{\prime}(m, n)\right]^{2}} \\
M S E=\frac{255^{2}}{10^{\frac{P S N R}{10}}}
\end{gathered}
$$

In formula (7), $f(m, n)$ as the original image pixels' value; $f^{\prime}(m, n)$ as image pixels' values after denoising; $M, N$ as rows and columns of image respectively.

In experiment, adding Gaussian white noise to the size of $256 \times 256$ of image Lena, then have de-noising process. Calculating PSNR and MSE of the image after the image have median filtering, Wiener filtering, wavelet threshold de-noising, wavelet and Wiener filtering respectively, the values of PSNR and MSE are shown in Table 1.

Comparing with the visual effect from Figures 2-7 and the data of PSNR and MSE in Table 1, we can see that the visual effect, peak signal to noise ratio and mean square error of the wavelet and Wiener filtering de-noising method are superior to median filtering algorithm, Wiener filtering and wavelet threshold algorithm. $t$ shows that the proposed wavelet and Wiener filtering de-noising algorithm not only can effectively suppress Gaussian white noise, but can keep the edge details of image clearly.

\section{Conclusion}

A common problem is that when suppressing noise at the same time edge details of the image will be damaged. Combining with the wavelet transform and Wiener filtering technology, an improved image de-noising method is proposed in this paper. The experimental results are analyzed from subjective and objective aspects, this improved method can get good de-noising effect at the same time avoiding the traditional problem of detail missing. 
Table 1. The values of PSNR and MSE after different filtering.

\begin{tabular}{|c|c|c|c|}
\hline \multicolumn{2}{|c|}{ Methods } & \multirow{2}{*}{$\begin{array}{c}\text { PSNR } \\
43.2840\end{array}$} & \multirow{2}{*}{$\begin{array}{c}\text { MSE } \\
3.0527\end{array}$} \\
\hline & $3 \times 3$ template & & \\
\hline \multirow[t]{3}{*}{ Median filtering } & $5 \times 5$ template & 41.0430 & 5.1430 \\
\hline & $7 \times 7$ template & 39.4042 & 7.4587 \\
\hline & $3 \times 3$ template & 45.2439 & 1.94440 \\
\hline \multirow[t]{2}{*}{ Wiener filtering } & $5 \times 5$ template & 43.7056 & 2.7703 \\
\hline & $7 \times 7$ template & 41.9419 & 4.1581 \\
\hline \multicolumn{2}{|c|}{ Wavelet threshold de-noising } & 67.9472. & 0.0104 \\
\hline \multicolumn{2}{|c|}{ Method is proposed in this paper } & 68.3349 & 0.0095 \\
\hline
\end{tabular}

From the de-noising effect, comparing this improved methods in this paper with classic methods, PSNR and $M S E$ are improved. This algorithm is better to protect the image edge information and de-noising effect is good, which is a stable and efficient image de-noising method.

\section{Acknowledgements}

This work is supported by the Teaching Research Project of Hubei Province under Grant 2014260.

\section{References}

[1] Chatterjee, P. and Milanfar, P. (2012) Patch-Based Near-Optimal Image De-Noising. IEEE Transactions on Image Processing, 21, 1635-1649.

[2] Milanfar, P. (2013) A Tour of Modern Image Filtering: New Insights and Methods, Both Practical and Theoretical. IEEE Signal Processing Magazine, 30, 106-128. http://dx.doi.org/10.1109/MSP.2011.2179329

[3] Ramani, S., Blu, T. and Unser, M. (2008) Monte-Carlo Sure: A Black-Box Optimization of Regularization Parameters for Generalde-Noising Algorithms. IEEE Transactions on Image Processing, 17, 1540-1554.

[4] Goossens, B., Pizurica, A. and Philips, W. (2009) Image de-Noising Using Mixtures of Projected Gaussian Scale Mixtures. IEEE Transactions on Image Processing, 18, 1689-1702.

[5] Zhang, M. and Gunturk, B.K. (2008) Multiresolution Bilateral Filtering for Image De-Noising. IEEE Transactions on Image Processing, 17, 2324-2333. http://dx.doi.org/10.1109/TIP.2008.2006658

[6] Zhang, Y.J. (2005) Image Engineering (II): Image Analysis. Tsinghua University Press, Beijing.

[7] Zou, J.H. and Li, X.X. (2010) A New De-Noising Algorithm for Low Illumination Color Image. Television Technology, 34, 14-16.

[8] Chen, W.F. (2004) Wavelet Analysis and Its Application in Image Processing. Science Press, Beijing, 156-157.

[9] Pan, Q., Zhang, L. and Meng, J.L. (2005) Wavelet Filtering Method and Application. Tsinghua University Press, Beijing.

[10] Ju, S.G., He, K. and Zhou, J.L. (2010) Image with Gauss Noise De-Noising Based on Neighborhood Characteristics. Journal of Sichuan University, 42, 139-144.

[11] Hu, X. and Zhang, L.J. (2010) Application of Adaptive Nonlinear Wavelet De-Noising in Gray Images. Television Technology, 34, 25-28.

[12] Donoho, D.L. (1995) De-Noising by Soft-Thresholding. IEEE Transactions on Information Theory, 41, 613-627. http://dx.doi.org/10.1109/18.382009

[13] Donoho, D.L. and Johnstone, I.M. (1995) Adapting to Unknown Smoothness via Wavelet Shrinkage. American Statistical Association, 90, 1200-1224.

[14] Wu, Y. and Wu, H.Y. (2010) An Adaptive Filtering Method for Image Denoising. Computer Engineering and Applications, 46, 168-170.

[15] Guo, S.X. and Tang, Y.J. (2008) The Popularization and Application of Image Processing Wiener Filter. Computer Engineering and Applications, 44, 178-180.

[16] Mohan, J. (2013) Image Denoising Based on Neutrosophic Wiener Filtering. Advances in Intelligent Systems and Computing, 177, 861-869. http://dx.doi.org/10.1007/978-3-642-31552-7_88 